\title{
Training on the Practical Approach to Lung Health: effect on drug prescribing in PHC settings in Jordan
}

K. Abu Rumman, ${ }^{1}$ S. Ottmani, ${ }^{2}$ N. Abu Sabra, ${ }^{1}$ S. Baghdadi, ${ }^{3}$ A. Seita ${ }^{3}$ and L. Blanc ${ }^{2}$

$$
\begin{aligned}
& \text { التدريب على الأسلوب العملي لصحة الرئة: أثر وصف الدواء في مواقع الرعاية الصحية الأولية في الأردن الردان }
\end{aligned}
$$

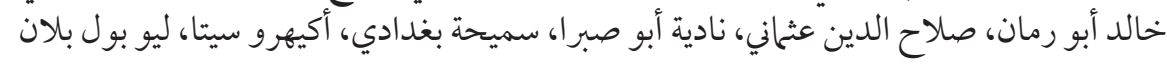

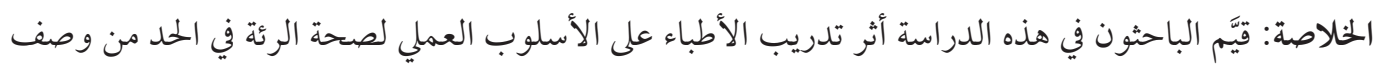

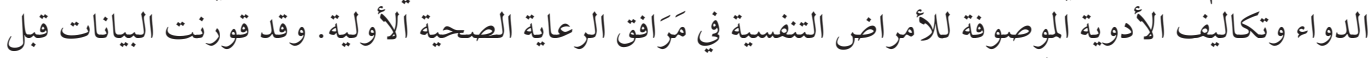

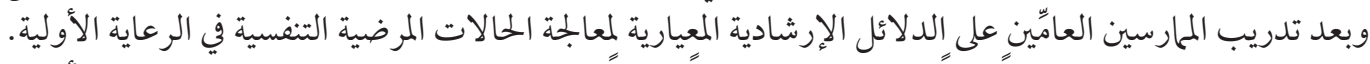

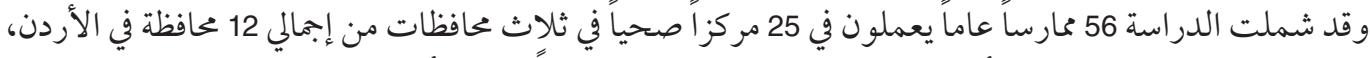

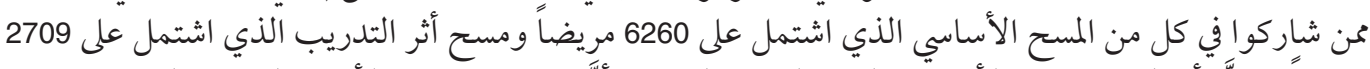

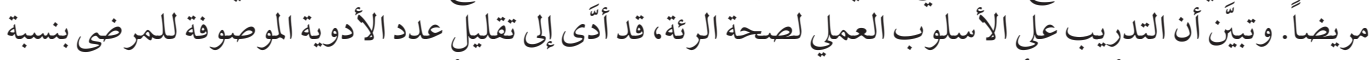

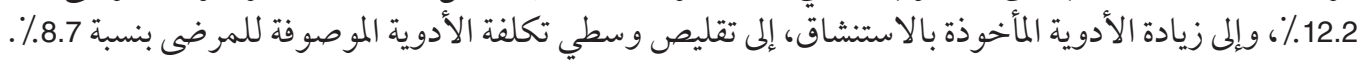

ABSTRACT This study assessed whether training physicians on the Practical Approach to Lung Health (PAL) reduces drug prescribing and the cost of drugs prescribed to respiratory patients in the primary health care setting. Data were compared before and after training general practitioners on standard guidelines for case management of respiratory conditions in primary care. A total of 56 general practitioners practising in 25 health centres in 3 out of 12 governorates of Jordan participated in both the baseline survey ( $n=6260$ respiratory patients) and the impact survey $(n=2709$ patients). Training in PAL decreased by $12.2 \%$ the number of drugs prescribed per patient, increased the prescription of inhaled medications and reduced the mean cost of a drug prescription per patient by $8.7 \%$.

Formation à l'approche pratique de la santé respiratoire : les effets sur la prescription de médicaments dans les structures de soins de santé primaires en Jordanie

RÉSUMÉ Cette étude visait à déterminer si le fait de former les médecins à l'approche pratique de la santé respiratoire (APSR) entraînait une réduction de la prescription de médicaments et diminuait le coût des médicaments prescrits aux patients souffrant d'affections respiratoires dans les structures de soins de santé primaires. Les données ont été comparées avant et après que les médecins généralistes aient reçu une formation sur les recommandations standard concernant la prise en charge des affections respiratoires dans le cadre des soins de santé primaires. Au total, 56 médecins généralistes exerçant dans 25 centres de santé situés dans 3 des 12 gouvernorats de Jordanie ont participé à l'étude de référence ( $n=6260$ patients souffrant d'affections respiratoires) et à l'étude d'impact ( $n=2709$ patients). La formation à l'APSR a entraîné une diminution de 12,2 \% du nombre de médicaments prescrits par patient, une augmentation de la prescription de médicaments inhalés et une réduction de $8,7 \%$ du coût moyen des prescriptions de médicaments par patient.

${ }^{1}$ National Tuberculosis Control Programme, Ministry of Health, Amman, Jordan.

${ }^{2}$ Stop TB Department, World Health Organization, Geneva, Switzerland (Correspondence to S. Ottmani: ottmanis@who.int).

${ }^{3}$ Stop TB Unit, Department of Communicable Diseases, World Health Organization Regional Office for the Eastern Mediterranean, Cairo, Egypt.

Received: 02/03/08; accepted: 27/05/08

المجلة الصحية لشرق المتوسط، منظمة الصحة العالمية، المجلد الخامس عشر، العدد ا، 9.ب. 


\section{Introduction}

Among patients aged 5 years and over who attend primary health care (PHC) facilities for any reason, $20 \%$ and more seek care for respiratory conditions in low- and middleincome countries $[1,2]$. Ten surveys carried out by the World Health Organization (WHO) in 76 PHC facilities in 9 developing countries showed that more than $95 \%$ of respiratory patients were prescribed at least 1 drug, and more than $75 \%$ of them may receive an antibiotic prescription [1]. The Practical Approach to Lung Health (PAL) is a patient-centred approach to diagnosis and treatment of common respiratory illnesses in PHC settings that has been developed by WHO within the framework of the Stop TB Strategy in order to contribute to health system strengthening [3]. To this end, PAL aims at improving the quality of respiratory case management as well as the efficiency of care delivery services for respiratory illnesses $[4,5]$. The PAL approach seeks to standardize service delivery through the development and implementation of clinical guidelines. It is also intended to coordinate not only among the different levels of health care but also among the national health programmes, including the TB control programme, and general health services $[6,7]$. Since 2001, many countries have introduced some form of PAL activities [7].

In March 2003, the national tuberculosis control programme (NTP) of Jordan initiated the process of PAL development in close collaboration with WHO. A national working group on PAL was established under the leadership of the NTP and included representatives from various departments of the Ministry of Health, academic institutions and the private medical sector. The national working group developed clinical guidelines that take into account the health context of Jordan [8] and training materials
[9]. The implementation of PAL was field tested using a study protocol developed by WHO [6].

One of the main objectives of this field testing was to assess whether the implementation of PAL reduces drug prescribing and its inherent costs in patients seeking care for respiratory symptoms in PHC facilities. This article reports the short-term impact of training physicians about PAL guidelines on drug prescribing observed during the field testing of PAL.

\section{Methods}

To assess the feasibility and short-term impact of training about PAL guidelines, the results of 2 surveys were compared. The first, or baseline, survey was undertaken before any training of general practitioners (GPs) and the second, or impact, survey after training GPS on PAL guidelines. To meet the requirements established in the WHO study protocol [6], both surveys were carried out in the same season and in the same PHC facilities and involved the same GPs. The same protocol was used in the process of data collection in the 2 surveys. The feasibility test was carried out in line with ethical standards of the NTP and the Ministry of Health of Jordan.

\section{Selection of the survey sites and GPs}

The surveys took place in the governorates of Amman, Balqa and Zarqa, which were selected based on the availability of qualified human resources and ease of access to facilitate supervision of the study. Out of the 75 public sector PHC centres in the 3 governorates, 25 were selected. The selection of these health facilities was based on 3 factors: the extent of primary care services that they provided to the local community; 
the availability of resources that would facilitate the collection of data of satisfactory quality; and patient access to the first level of referral above PHC. Out of the 63 GPs who were practising in these 25 centres, 7 could not participate in all of the phases required to carry out a feasibility test of PAL (baseline survey, training on PAL and impact survey). Therefore, 56 of the 63 GPs $(89 \%)$ participated in both surveys.

\section{Eligibility of the participants and case definitions}

Any patient aged 5+ years who sought care for at least 1 respiratory symptom in any of the selected PHC centres during the study period was eligible to be enrolled in the surveys. The complaint of respiratory symptom(s) should originate spontaneously from the patient and not be suggested to her/ him at the time of the visit. A respiratory symptom was any clinical manifestation generated from the upper respiratory tract (e.g. ear pain, ear discharge, ear pruritis, nasal obstruction, nasal discharge, nasal pruritis, postnasal drip, sneezing, epistaxis, dysphonia, sore throat, painful swallowing, cervical tumour, etc.) or from or the lower respiratory tract (e.g. cough, short breathing, expectoration, wheezing, haemoptysis, chest pain, etc.). The respiratory symptoms could be isolated or accompanied by other symptoms such as fever, fatigue, weight loss, cyanosis, dyspepsia, etc.

Suspected cases of TB were identified using the definition of the NTP (i.e. cough and expectoration for more than 14 days). The other respiratory conditions were labelled according to disease categories proposed in the study protocol.

\section{Data collection}

The GPs involved in the study registered specific information on every enrolled patient in a registry form. This information included survey identification number, age, sex, clinical symptoms, probable diagnosis and management decision (prescription for treatment and/or referral to higher level of health care). The diagnosis was strictly reported in line with the categories listed in the study protocol.

For each patient enrolled in the study, the survey identification number specified in the registry form was systematically registered in the clinical record, drug prescription form, treatment card and any patient health documents. When a patient was prescribed drugs, a copy of that prescription was kept on the data collection site. The cost of the drug prescription was established on the basis of the prices used in private pharmacies. This cost was specified for every study participant who was prescribed drugs as if she/he had purchased the prescribed drugs in a private pharmacy.

\section{Baseline survey}

One week before the baseline survey, 56 GPs were trained for 1 day about using the study register and forms in order to collect the data needed in a standardized manner. Nine coordinators ensured the supervision and monitoring of the implementation of the survey and the process of data collection. To carry out the baseline survey, the eligibility criteria of the study participants, the definitions and the process of data collection were used as described above. The recruitment of the respiratory patients for the baseline survey took place in the $25 \mathrm{PHC}$ facilities from 12 to 16 December 2004.

\section{Training on PAL guidelines}

After the baseline survey was completed, the 56 participating GPs were trained on the use of the national PAL guidelines from 23 to 27 January 2005. The GPs were divided into 3 groups of 23, 21 and 12 GPs who were trained during the same week but sep- 
arately. Each group was trained over 4 days by facilitators, including 2-3 of the 9 coordinators and at least 1 senior staff from the NTP and 1 senior chest specialist. The same training materials and procedures were used for the 3 groups. The training included: plenary sessions on the basic elements of PAL; respiratory care and the health management information system; case studies with simulation exercises that covered the whole content of the guidelines; a practical session on the use of the peak-flow meter and inhalation chamber; a session with role play; and practical demonstrations of respiratory case management using PAL guidelines in PHC facilities.

\section{Impact survey}

After training on PAL guidelines, the impact survey was carried out from 6 to 10 February 2005 in the same 25 PHC facilities. As in the baseline survey, the 56 GPs used the same eligibility criteria to enrol study participants, as well as the same definitions and the same procedures to collect information. However, the respiratory patients who were enrolled in the impact survey were managed as specified in the PAL guidelines. This was the key difference from the baseline survey.

\section{Statistical analysis}

A data entry programme was adapted specifically for these surveys, using Epi-Info, version $6.04 \mathrm{~b}$. The data analysis involved studying the distribution of the variables registered in each survey and the drugs prescribed to respiratory patients, particularly antibiotics, bronchodilators, corticosteroids and adjuvant drugs; the cost of drug prescriptions was assessed in each survey. The findings were compared between the baseline and impact surveys. The Student $t$ test was used in the comparison of 2 means, and the chi-squared test for comparison of proportions. The Fisher exact test was used when the expected number was below 5 in any $2 \times 2$ contingency table. The MannWhitney test was used in the comparison of 2 non-normal distributions of continuous variables. A statistical difference was considered significant when the $P$-value was below $5 \%$.

\section{Results}

\section{Characteristics of respiratory patients}

In the baseline survey, 6260 respiratory patients were enrolled and in the impact survey there were 2709. The sex distribution of the study participants was not significantly different between the 2 surveys ( $P$ $>0.05)$. However, the mean age of respiratory patients for both sexes was lower in the baseline survey (Table 1). The duration of symptoms, from their onset to the patient's visit to the PHC facility, was significantly different between the baseline survey [mean 4.0 (SD 12.0) days; median 2.0 days; mode 2.0 days] and the impact survey [mean 4.8 (SD 13.5) days; median 3.0 days; mode 2 days] (Mann-Whitney test: $P<0.001$ ).

The proportion of patients with at least 1 concomitant condition or disease, and the proportion who visited a health facility for respiratory symptoms at least once within the previous month was significantly different between the 2 surveys. Cough, fever, nasal discharge, sore throat and expectoration were the most frequently reported symptoms in both surveys (Table 2).

Patients with acute respiratory infection (ARI) accounted for a very high proportion in both surveys ( $90 \%$ or more); almost twothirds of the patients in each of the 2 surveys had an acute upper respiratory infection. The proportion with chronic respiratory disease (CRD) was significantly higher in the impact survey (Table 3). 
Table 1 Sex distribution and mean age of respiratory patients aged $\geq \mathbf{5}$ years enrolled in the baseline and impact surveys of training physicians on the Practical Approach to Lung Health

\begin{tabular}{|c|c|c|c|c|c|}
\hline \multirow[t]{2}{*}{ Variable } & \multicolumn{2}{|c|}{ Baseline survey } & \multicolumn{2}{|c|}{ Impact survey } & \multirow[t]{2}{*}{ Statistical tests } \\
\hline & No & $\%$ & No. & $\%$ & \\
\hline \multicolumn{6}{|l|}{$\begin{array}{l}\text { Patients with respiratory } \\
\text { symptoms }\end{array}$} \\
\hline Female & 3340 & 53.4 & 1427 & 52.7 & \\
\hline Male & 2920 & 46.6 & 1282 & 47.3 & $\chi^{2}=0.35 ; P=0.554$ \\
\hline Total & 6260 & 100.0 & 2709 & 100.0 & \\
\hline $\begin{array}{l}\text { Age of respiratory } \\
\text { patients (years) }\end{array}$ & \multicolumn{2}{|c|}{ Mean (SD) } & \multicolumn{2}{|c|}{ Mean (SD) } & \\
\hline Female & \multicolumn{2}{|c|}{$26.5(17.8)^{a}$} & \multicolumn{2}{|c|}{$31.5(18.3)^{b}$} & $t=8.75 ; P<0.001$ \\
\hline Male & \multicolumn{2}{|c|}{$22.4(17.8)^{\mathrm{a}}$} & \multicolumn{2}{|c|}{$29.4(18.8)^{b}$} & $t=11.46 ; P<0.001$ \\
\hline Total & \multicolumn{2}{|c|}{$24.6(17.9)$} & \multicolumn{2}{|c|}{$30.5(18.5)$} & $t=14.11 ; P<0.001$ \\
\hline
\end{tabular}

${ }^{a}$ Comparison of mean age of females and males in the baseline study $(\mathrm{t}=9.17 ; \mathrm{P}<0.001)$.

${ }^{b}$ Comparison of mean age of females and males in the impact study $(\mathrm{t}=3.00 ; \mathrm{P}<0.01)$.

$S D=$ standard deviation.

\begin{tabular}{|c|c|c|c|c|c|}
\hline \multirow[t]{2}{*}{ Variable } & \multicolumn{2}{|c|}{$\begin{array}{l}\text { Baseline survey } \\
\qquad(n=6260)\end{array}$} & \multicolumn{2}{|c|}{$\begin{array}{l}\text { Impact survey } \\
(n=2709)\end{array}$} & \multirow[t]{2}{*}{$P$-value } \\
\hline & No. & $\%$ & No. & $\%$ & \\
\hline \multicolumn{6}{|l|}{ Prior visits to any health facility } \\
\hline $\begin{array}{l}\text { Concomitant health condition or } \\
\text { disease }\end{array}$ & 991 & 15.8 & 559 & 20.6 & $<0.001$ \\
\hline \multicolumn{6}{|l|}{ Sign/symptom } \\
\hline Cough & 4671 & 74.6 & 1805 & 66.6 & $<0.001$ \\
\hline Fever & 3842 & 61.4 & 1571 & 58.0 & $<0.01$ \\
\hline Nasal discharge & 2041 & 32.6 & 678 & 25.0 & $<0.001$ \\
\hline Sore throat & 1360 & 21.7 & 778 & 28.7 & $<0.001$ \\
\hline Expectoration & 1061 & 16.9 & 522 & 19.3 & $<0.01$ \\
\hline Short breathing and/or wheezing & 391 & 6.2 & 210 & 7.8 & $<0.01$ \\
\hline Chest pain & 371 & 5.9 & 174 & 6.4 & 0.366 \\
\hline Wheezing & 268 & 4.3 & 217 & 8.0 & $<0.001$ \\
\hline Ear pain & 217 & 3.5 & 155 & 5.7 & $<0.001$ \\
\hline Painful swallowing & 156 & 2.5 & 23 & 0.8 & $<0.001$ \\
\hline Dysphonia & 78 & 1.2 & 38 & 1.4 & 0.550 \\
\hline Cervical lymph node & 50 & 0.8 & 17 & 0.6 & 0.390 \\
\hline Haemoptysis & 38 & 0.6 & 11 & 0.4 & 0.236 \\
\hline Ear discharge & 37 & 0.6 & 30 & 1.1 & $<0.01$ \\
\hline Other symptoms & 1582 & 25.3 & 653 & 24.1 & 0.241 \\
\hline
\end{tabular}

المجلة الصحية لشرق المتوسط، منظمة الصحة العالمية، المجلد الخامس عشر، العدد (، 9. ب. 
Table 3 Distribution of respiratory conditions diagnosed among respiratory patients enrolled in the baseline and impact surveys

\begin{tabular}{lrrrrr}
\hline $\begin{array}{l}\text { Respiratory } \\
\text { condition }\end{array}$ & $\begin{array}{r}\text { Baseline survey } \\
(\boldsymbol{n}=\mathbf{6 2 6 0})\end{array}$ & \multicolumn{2}{c}{ Impact survey $(\boldsymbol{n}=\mathbf{2 7 0 9})$} & P-value \\
\hline ARI & 5835 & 93.2 & 2419 & 89.3 & \\
AURI & 4003 & 63.9 & 1741 & 64.2 & $<0.001$ \\
ALRI & 1832 & 29.3 & 678 & 25.0 & 0.771 \\
CRD & 378 & 6.0 & 263 & 9.7 & $<0.001$ \\
High suspicion of TB & 29 & 0.5 & 20 & 0.7 & 0.001 \\
Other condition & 18 & 0.3 & 7 & 0.3 & 0.105 \\
\hline
\end{tabular}

$A R I=$ acute respiratory infection; $A U R I=$ acute upper respiratory infection; $A L R I=$ acute lower respiratory infection; $C R D=$ chronic respiratory disease $; B=$ tuberculosis.

\section{Changes in drug prescribing}

In both surveys, $97 \%$ of the respiratory patients were prescribed drugs. The number of drugs prescribed per patient decreased by $12.2 \%$ between the baseline and impact surveys. Among the patients who received a drug prescription, the proportion who were prescribed antibiotics decreased by $15.9 \%$ in the impact survey. This decrease was observed in ARI and CRD patients (Table 4).

The prescription of bronchodilator drugs was $<10 \%$ among patients who were prescribed drugs in both surveys. However, the prescription of bronchodilator drugs increased significantly for inhaled $\beta$-agonists and decreased for the other forms of bronchodilators in the impact survey (Table 4).

The prescription of corticosteroids increased significantly, with an increase in prescription for inhalation use; the prescription of injectable corticosteroids decreased but not statistically significantly (Table 4).

In the impact survey, the proportion of patients who were prescribed antihistamine drugs, non-steroidal anti-inflammatory drugs, expectorants or bronchial fluidifiers and vitamins significantly decreased, while that of patients who were prescribed paracetamol and nasal decongestants significantly increased (Table 4).

\section{Changes in cost of prescriptions}

The mean cost of the prescription of any drug per patient was reduced by $8.7 \%$ between the 2 surveys $(P<0.05)$. This was particularly significant for antibiotics and adjuvant drugs. However, the mean cost of antibiotic prescriptions per patient who was prescribed antibiotics did not change between the 2 surveys, whereas the mean cost of adjuvant drug prescriptions decreased significantly among patients who received adjuvant drug prescriptions (Table 5).

Among patients who were prescribed bronchodilators, the mean cost of bronchodilators was significantly higher in the impact survey.

Furthermore, among patients prescribed corticosteroids, the mean cost of corticosteroids decreased in the impact survey, but not statistically significantly (Table 5 ). However, among the 43 and 51 respiratory patients who were prescribed inhaled corticosteroids in the baseline and impact surveys respectively, the mean cost per patient of the prescription of this inhaled medication decreased by $21.5 \%$, from 3.8 Jordanian dinars (SD 2.3) in the baseline survey to 3.0 Jordanian dinars (SD 1.7) in the impact survey $(P<0.05)$. 


\begin{tabular}{|c|c|c|c|c|c|c|}
\hline \multirow[t]{2}{*}{$\begin{array}{l}\text { Drug prescription } \\
\text { No. of drugs per patient }\end{array}$} & \multicolumn{2}{|c|}{$\begin{array}{c}\text { Baseline survey } \\
(n=6260) \\
\text { Mean (SD) }\end{array}$} & \multicolumn{2}{|c|}{$\begin{array}{l}\text { Impact survey } \\
(n=2709) \\
\text { Mean (SD) }\end{array}$} & $\begin{array}{c}\% \\
\text { change }^{a}\end{array}$ & $P$-value \\
\hline & \multirow{2}{*}{\multicolumn{2}{|c|}{$2.55(0.77)$}} & \multirow{2}{*}{\multicolumn{2}{|c|}{$2.24(0.76)$}} & & \\
\hline prescribed drugs & & & & & -12.2 & $<0.001$ \\
\hline \multicolumn{7}{|l|}{ No. of antibiotics prescribed } \\
\hline \multirow[t]{2}{*}{ per patient prescribed drugs } & \multicolumn{2}{|c|}{$0.90(0.15)$} & \multicolumn{2}{|c|}{$0.77(0.24)$} & -15.0 & $<0.001$ \\
\hline & No. & $\%$ & No. & $\%$ & & \\
\hline $\begin{array}{l}\text { No. of patients prescribed } \\
\text { drugs/total no. of patients }\end{array}$ & $6077 / 6260$ & 97.1 & $2630 / 2709$ & 97.1 & 0.0 & 0.985 \\
\hline $\begin{array}{l}\text { No. of patients prescribed } \\
\text { antibiotics/no. prescribed } \\
\text { drugs }\end{array}$ & & & & & & \\
\hline $\begin{array}{l}\text { drugs } \\
\text { No. of patients prescribed } \\
\text { antibiotics/no. prescribed } \\
\text { drugs for: }\end{array}$ & $5304 / 6077$ & 87.3 & $1931 / 2630$ & 73.4 & -15.9 & $<0.001$ \\
\hline AURI & $3399 / 3905$ & 87.0 & $1299 / 1722$ & 75.4 & -13.3 & $<0.001$ \\
\hline ALRI & $1635 / 1788$ & 91.4 & $529 / 658$ & 80.4 & -12.0 & $<0.001$ \\
\hline CRD & $259 / 363$ & 71.3 & $101 / 245$ & 41.2 & -42.2 & $<0.001$ \\
\hline High suspicion of TB & $4 / 7$ & 57.1 & $0 / 1$ & 0.0 & -100.0 & $1.0^{\mathrm{b}}$ \\
\hline Other & $7 / 14$ & 50.0 & $2 / 4$ & 50.0 & 0.0 & $1.0^{\mathrm{b}}$ \\
\hline $\begin{array}{l}\text { No. of patients prescribed } \\
\text { a specific medication/no. } \\
\text { prescribed drugs }(n=6077) \text { : }\end{array}$ & & & & & & \\
\hline Any bronchodilator & 261 & 4.3 & 163 & 6.2 & +44.2 & $<0.001$ \\
\hline+ inhaled $\beta$-agonist & 109 & 1.8 & 121 & 4.6 & +155.0 & $<0.001$ \\
\hline+ other form of $\beta$-agonist & 146 & 2.4 & 42 & 1.6 & -33.3 & $<0.05$ \\
\hline + theophylline & 12 & 0.2 & 2 & 0.1 & -62.0 & $0.253^{b}$ \\
\hline Any corticosteroid & 73 & 1.2 & 71 & 2.7 & +125.0 & $<0.001$ \\
\hline + bronchial inhalation & 36 & 0.6 & 42 & 1.6 & +166.7 & $<0.001$ \\
\hline + nasal inhalation & 12 & 0.2 & 13 & 0.5 & +150.0 & $<0.05$ \\
\hline+ tablet & 12 & 0.2 & 18 & 0.7 & +250.0 & $<0.001$ \\
\hline + injection & 18 & 0.3 & 5 & 0.2 & -33.3 & 0.670 \\
\hline Paracetamol & 4795 & 78.9 & 1986 & 75.5 & +4.3 & $<0.001$ \\
\hline Nasal decongestant & 170 & 2.8 & 129 & 4.9 & +75.0 & $<0.001$ \\
\hline Antihistamine & 2570 & 42.3 & 608 & 23.1 & -45.4 & $<0.001$ \\
\hline NSAID & 845 & 13.9 & 213 & 8.1 & -41.7 & $<0.001$ \\
\hline Expectorant/fluidifier & 504 & 8.3 & 137 & 5.2 & -37.3 & $<0.001$ \\
\hline Vitamins & 24 & 0.4 & 5 & 0.2 & -50.0 & $<0.05$ \\
\hline
\end{tabular}

${ }^{a}$ Between baseline and impact surveys.

${ }^{b}$ Fisher exact test.

$S D=$ standard deviation

$A U R I=$ acute upper respiratory infections; $A L R I=$ acute lower respiratory infections; $C R D=$ chronic respiratory disease; $T B=$ tuberculosis; NSAID = non-steroidal anti-inflammatory drug. 


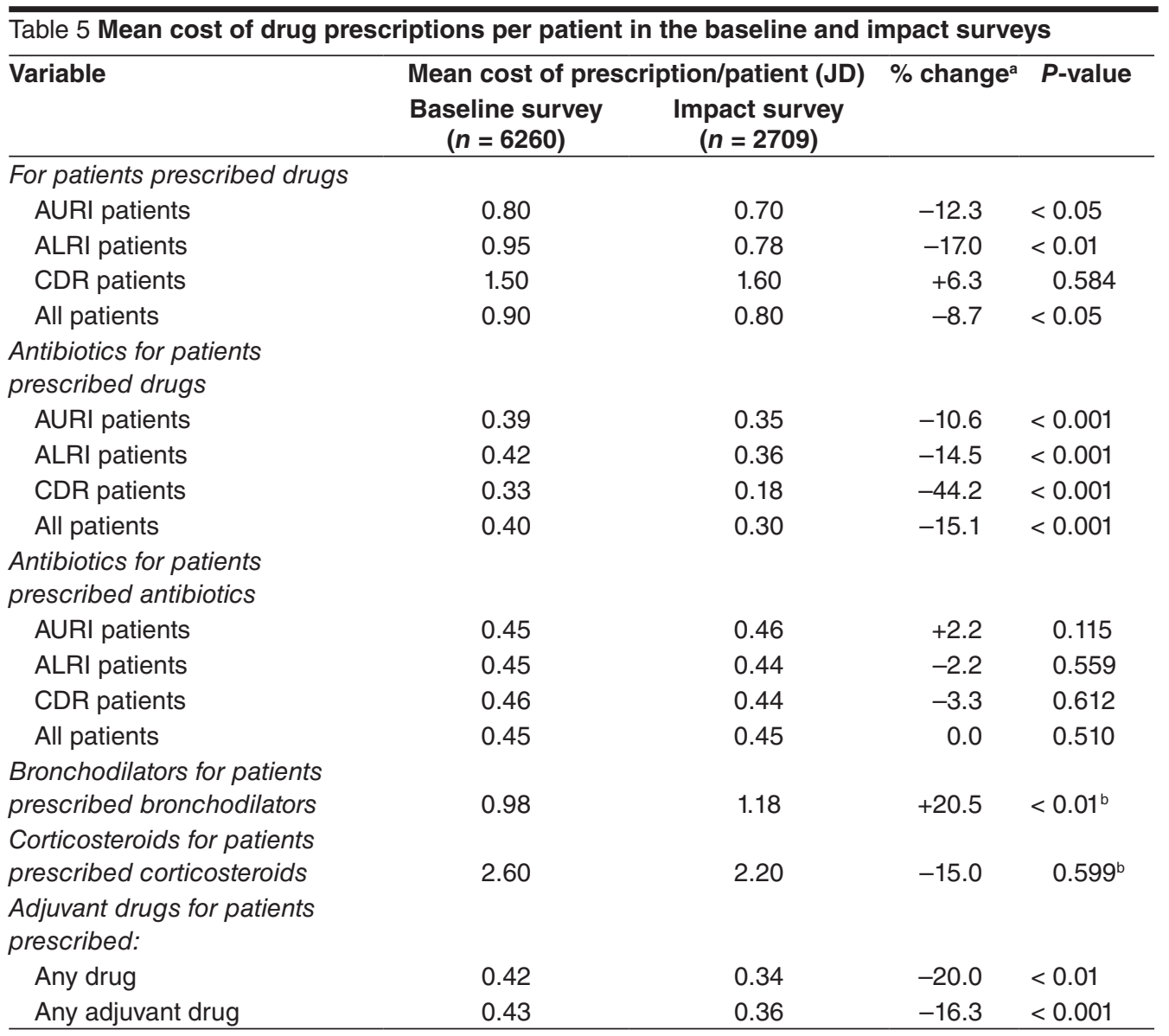

${ }^{a}$ Between baseline and impact surveys.

${ }^{b}$ Mann-Whitney test for non-normal distribution.

$J D=$ Jordanian dinar; $A U R I=$ acute upper respiratory infection; $A L R I=$ acute lower respiratory infection; $C R D=$ chronic respiratory disease;

\section{Discussion}

To meet the requirements established by WHO for PAL feasibility testing [6], the same study protocol was used in the baseline and impact surveys. Also, both surveys took place in the same season, within a 7-week interval in winter, in the same PHC facilities and with the same GPs, in order to ensure comparability between the data sets of the 2 surveys. As the 56 GPs were the same in both surveys, it is expected that their practice in case management of respiratory patients would be influenced only by the training on PAL guidelines.

However, the number of respiratory patients enrolled in the baseline survey was considerably higher than in the impact survey. This important difference in patient recruitment was due to the occurrence of a snowstorm in the 3 study governorates 
during the week of the impact survey. As a result, many economic and social activities decreased in that week, including disruptions to transportation as well as decreased attendance at PHC facilities by patients. On average, clusters of 112 and 48 respiratory patients were managed by every GP in the baseline and impact surveys respectively. Patients included in the same cluster were likely to be managed in a similar manner by the GP, resulting in a clustering effect that may have influenced the results in both surveys. However, given that the average number of study participants included in a cluster was 2.3 times higher in the baseline survey than in the impact survey, the clustering effect is likely to be stronger in the first survey than in the second. Because of this, some degree of distortion was probably introduced in the comparability between the data sets of the 2 surveys. It is not clear how this may have influenced the findings of this study.

The sex distribution was not significantly different between the 2 surveys, but the patients enrolled in the impact survey were older and tended to have more concomitant diseases. The distribution of respiratory illnesses was fairly similar between the 2 surveys. However, in comparison with the baseline study, the proportion of patients with CRDs was higher in the impact survey, while the proportion of those with acute lower respiratory infections was lower. It is not clear whether this shift in the distribution was associated with the training on PAL guidelines or the higher proportion of study participants with a concomitant disease in the impact survey. It is not known either to what extent the limitations in the comparability between the participants of the 2 surveys may have influenced this shift.

The comparison of the findings between the baseline and impact surveys suggests that training on PAL guidelines had an im- pact in decreasing drug prescription through a reduction in prescriptions for antibiotics and adjuvant drugs. There was also a reduction in the mean cost of drug prescriptions per patient after training on PAL. The cost reduction in the impact survey seemed to be related not only to the decrease in the prescriptions of antibiotics and adjuvant drugs but also to a decrease in the cost of adjuvant drug prescriptions in patients who were prescribed such medications. Similar results were reported in other countries where PAL methods have been initiated. For instance, the average cost of drug prescriptions per patient was reduced by $13.6 \%$ in Algeria (N. Zidouni, personal communication), 32.2\% in Bolivia [2], 32.4\% in Kyrgyzstan [4], $18.1 \%$ in Morocco [4], 26.2\% in the Syrian Arab Republic (F. Maamri, personal communication), $19.3 \%$ in Tunisia (A. Ben Kheder, personal communication) and by 2.5 rupees in Nepal [10].

Our study indicates that training on PAL is likely to increase the prescription of inhaled bronchodilators and inhaled corticosteroids and tends to decrease the prescription of the other formulations of these 2 categories of drugs. Similar findings were reported for both types of medication in Kyrgyzstan (N. Brimkulov, personal communication), the Syrian Arab Republic (F. Maamri, personal communication) and Tunisia (A. Ben Kheder, personal communication); for inhaled beta-agonists in Morocco (N. Bencheikh, personal communication); and for inhaled corticosteroids in South Africa [11].

The mean cost of corticosteroid prescriptions per patient who was prescribed this medication tended to decrease after GPs were trained on PAL methods; moreover, this decrease was significant in patients who were prescribed inhaled corticosteroids. Jordan has a well-functioning Essential Drugs Programme implemented in PHC;

المجلة الصحية لشرق المتوسط، منظمة الصحة العالمية، المجلد الخامس عشر، العدد (،9.ب. 
a wide range of antiasthmatic drugs and brands of corticosteroids for inhalation use are included in this programme. Inhaled beclomethasone is the corticosteroid for inhalation use that is specified in the PAL guidelines developed in Jordan, and is included in a generic version in the Essential Drugs Programme. Inhaled beclomethasone in the generic version is usually one of the cheapest inhaled corticosteroids. Therefore, training physicians on PAL not only increased the prescription of corticosteroids for inhalation use but also promoted their cheapest formulation; this suggests that the PAL approach can facilitate making inhaled corticosteroids available for a wider range of respiratory patients in PHC. Findings from Kyrgyzstan (N. Brimkulov, personal communication), the Syrian Arab Republic (F. Maamri, personal communication) and Tunisia (A. Ben Kheder, personal communication) showed that training physicians on PAL increased the average cost of corticosteroids per patient because of the high price of these drugs in their inhaled presentation. Inhaled corticosteroids are still not accessible for many patients because of their non-availability and/or prohibitive pricing in many developing countries [12,13].

Despite the limitations of this study, the findings suggest that implementation of the PAL approach in the PHC setting is likely to reduce drug prescribing for antibiotics and adjuvant drugs in respiratory patients, to increase the use of inhalation medications, which is highly recommended in the management of asthma and chronic obstructive pulmonary disease $[14,15]$, and to decrease the cost of drugs prescribed to respiratory patients in PHC. These results are in line with the findings of studies on PAL carried out in other country settings. However, the experience of PAL implementation in many countries has not been fully reported. More documentation is needed on the effects, particularly its impact on costeffectiveness of respiratory care services, TB case detection and strengthening the health system.

\section{Acknowledgements}

The study was supported by a grant from the World Health Organization.

The authors are indebted to the local survey medical coordinators for their collaboration and help: Dr Ezzat Abu Hashem, Dr Miassar Zenddah, Dr Sae'b Khashman, and Dr Khaleel Hanash in Amman Governorate; Dr Jamal El-Dabbas and Dr Emad Husaini in Balqa Governorate; Dr Hesham Sae'di, Dr Seyam Te'meh and Dr Derar Al-Rawsh in Zarqa governorate.

The authors gratefully acknowledge the valuable input and collaboration of Mrs Tejan Ali Elayan and Mrs Rasha Ali Oudeh from the Central Unit of the National TB Programme of Jordan.

\section{References}

1. Practical Approach to Lung Health. Respiratory care in primary care settings: a survey in 9 countries. Geneva, World Health Organization, 2004 (WHO/HTM/ TB/2004.333).

2. Camacho $M$ et al. Results of PAL feasibility test in primary health care facilities in four regions of Bolivia. International journal of tuberculosis and lung disease, 2007, 11:1246-52.

3. The Stop TB Strategy: building on and enhancing DOTS to meet the TB-related Millennium Development Goals. Geneva, World Health Organization, 2006 (WHO/ HTM/TB/2006.368). 
4. A primary health care strategy for the integrated management of respiratory conditions in people of five years of age and over. Geneva, World Health Organization, 2005 (WHO/HTM/TB/2005.351).

5. Murray JF, Pio A, Ottmani S. PAL: a new and practical approach to lung health. International journal of tuberculosis and lung disease, 2006, 10:1188-91.

6. Practical Approach to Lung Health: manual to initiate PAL implementation. Geneva, World Health Organization, 2008.

7. Evaluation of the Practical Approach to Lung Health. Report of meeting held on 18 and 19 June 2007. Geneva, World Health Organization, 2007 (WHO/HTM/ TB/2008.396).

8. Practical guide for the management of patients with respiratory symptoms in primary health care facilities in Jordan. Amman, Jordan, Ministry of Health, Division of Tuberculosis and Respiratory Diseases, 2003.

9. Practical approach to lung health training material. Amman, Jordan, Ministry of Health, Division of Tuberculosis and Respiratory Diseases, 2003.

10. Shrestha $\mathrm{N}$ et al. Practical Approach to Lung Health in Nepal: better prescribing and reduction of cost. Tropical medicine and international health, 2006; 11:76572.

11. Fairall LR et al. Effect of educational outreach to nurses on tuberculosis case detection and primary care of respiratory illness: pragmatic cluster randomised controlled trial. British medical journal, 2005, 331:750-4.

12. Watson JP, Lewis RA. Is asthma treatment affordable in developing countries? Thorax, 1997, 52:605-07.

13. Aït-Khaled N, Enarson D, Bousquet J. Chronic respiratory diseases in developing countries: the burden and strategies for prevention and management. Bulletin of the World Health Organization, 2001 , 79:971-9.

14. GINA report: global strategy for asthma management and prevention, updated 2007. Global Initiative for Asthma [website] (http://www.ginasthma.org, accessed 6 July 2008).

15. Global strategy for the diagnosis, management and prevention of chronic obstructive pulmonary disease, updated 2007. Global Initiative for Chronic Obstructive Lung Disease [website] (http://www.goldcopd.org, accessed 6 July 2008). 